\title{
MORBIMORTALIDADE DA COLECISTECTOMIA EM PACIENTES IDOSOS, OPERADOS PELAS TÉCNICAS LAPAROTÔMICA, MINILAPAROTÔMICA E VIDEOLAPAROSCÓPICA
}

\section{Morbimortality of elderly patients submitted to cholecystectomy by laparotomy, minilaparotomy or videolaparoscopy}

\author{
José Guilherme MINOSSI, Hémerson César PICANÇO, Marcelo Antônio de CARVALHO, \\ Paulo Roberto Vasconcelos PAULUCCI, Soraya VENDITES
}

ABCDDV/535

\begin{abstract}
Minossi JG, Picanço HC, Carvalho MA, Paulucci PRV, Vendites S. Morbimortalidade da colecistectomia em pacientes idosos, operados pelas técnicas laparotômica, minilaparotômica e videolaparoscópica. ABCD Arq Bras Cir Dig 2007; 20(2):93-6.

RESUMO - Racional - A colelitíase é uma das doenças mais freqüentes do aparelho digestivo, acometendo $20 \%$ da população adulta. A idade tem sido considerada importante fator preditivo de complicações após a colecistectomia. Objetivo - Avaliar a morbimortalidade da colecistectomia em pacientes idosos operados por técnica laparotômica, minilaparotômica e videolaparoscópica. Método - Foram analisados retrospectivamente 557 pacientes submetidos à colecistectomia associada ou não a outros procedimentos sobre as vias biliares no período de julho de 1985 a dezembro de 2003. Desses, 152 pacientes (27,3\%) tinham 60 ou mais anos e $120(79 \%)$ eram do sexo feminino. Noventa e dois doentes foram operados por laparotomia, 46 por videolaparoscopia e 14 por minilaparotomia. Resultados - As complicações ocorreram com mais freqüência e gravidade nos pacientes mais idosos e operados por laparotomia, sendo que estes apresentaram maior índice de infecção urinária, da ferida cirúrgica e maior tempo de permanência hospitalar. Houve três casos de óbito, sendo dois após laparotomia e outro após videolaparoscopia, todos acima de 70 anos de idade. Conclusãa - A idade é importante fator preditivo de complicações após a colecistectomia, tanto pela incidência aumentada da doença do trato biliar complicada, como pela maior morbimortalidade da operação no idoso, em decorrência de doenças associadas.

DESCRITORES - Colecistectomia. Laparoscopia. Colelitíase. Mortalidade.
\end{abstract}

\section{INTRODUÇÃO}

A colecistolitíase é uma das doenças mais freqüentes do aparelho digestivo, acometendo $20 \%$ da população adulta $^{1}$

Desde sua primeira descrição em 1882, por Langenbruch, a colecistectomia se mantém como o tratamento de escolha para a colecistolitíase ${ }^{2}$. Desde essa época os cirurgiões tentam diminuir a morbidade, mortalidade e tempo de internação que é muito variável na literatura.

A colecistectomia por minilaparotomia, divulgada por Goco e Chambers em $1982^{3}$ e a colecistectomia videolaparoscópica, por Mouret em 1987, diminuíram o trauma cirúrgico e o período de internaçã $0^{4,5}$.

A idade tem sido considerada um importante fator preditivo de complicações após colecistectomia pela incidência aumentada da doença no trato biliar e pela maior morbimortalidade da operação no idoso, em decorrência das doenças associadas comuns na idade ${ }^{6,7}$.

O presente trabalho teve por objetivo avaliar os aspec-

Trabalho realizado na Santa Casa de Misericórdia de Cerqueira César, SP, Brasil.

Endereço para correspondência: José Guilherme Minossi, e-mail: jminossi@fmb.unesp.br tos gerais, morbidade e mortalidade das colecistectomias realizadas pelas vias laparotômica, minilaparotômica e videolaparoscópica em pacientes idosos, comparados com outros de menor idade.

\section{MÉTODOS}

Foram analisados retrospectivamente as fichas dos pacientes submetidos à colecistectomia associada ou não a outros procedimentos sobre as vias biliares, em caráter eletivo ou de urgência no período de agosto de 1985 a dezembro de 2003.

Dados relativos ao sexo, idade, operações realizadas, tempo de internação, complicações intra e pós-operatórias foram consideradas até o $30^{\circ}$ dia do procedimento. Considerou-se pacientes idosos aqueles com 60 ou mais anos.

Todos tinham ficha padrão que era preenchida após a alta hospitalar e completada na alta definitiva, ao final do primeiro mês.

De 1985 a 1991 todos os pacientes foram operados por técnica laparotômica. Em 1991 introduziu-se a colecistectomia por minilaparotomia, quando foram operados 60 pacientes até 1995, sendo 14 idosos. Neste ano iniciou-se a colecistectomia videolaparoscópica e, a partir de então, 
191 pacientes foram operados por esta via, sendo 46 idosos. As operações por minilaparotomia foram abandonadas, mas mantidas as laparotômicas, quando não indicada a via laparoscópica. Do total de laparotomias realizadas (306), 92 ocorreram em idosos.

Antibioticoterapia não foi utilizada rotineiramente, embora a maioria dos pacientes tenha recebido antibiótico profilático, que nos anos 80 foi cloranfenicol, posteriormente ampicilina e recentemente cefalotina em monodose na indução anestésica ou por no máximo 24 horas após a operação. Antibioticoterapia curativa foi utilizada somente quando havia infecção e por períodos de 5 a 10 dias, dando-se preferência às cefalosporinas de segunda geração (cefoxitina) associadas ou não a aminoglicosídeos.

Todas as intervenções foram feitas pelo mesmo grupo.

\section{RESULTADOS}

Durante o período analisado foram operados 557 pacientes, 456 (81,8\%) eram do sexo feminino e 101 (18,2\%) do sexo masculino. A idade variou de 8 à 92 anos, sendo que 152 pacientes $(27,3 \%)$ tinham 60 ou mais anos. Destes, $120(79 \%)$ eram do sexo feminino. Dos 152 pacientes considerados idosos, 92 foram operados por técnica lapatorômica, 46 por via laparoscópica e 14 por minilaparotomia (Tabela 1).

TABELA 1 - Colecistectomias realizadas por via laparotômica, videolaparoscopia e minilaparotomia nas faixas etárias acima e abaixo de 60 anos

\begin{tabular}{lccc}
\hline Tipo de procedimento & Idade $<\mathbf{6 0}$ & Idade $\geq \mathbf{6 0}$ & Total \\
\hline Colecistectomia por laparotomia & 214 & 92 & 306 \\
Colecistectomia videolaparoscópica & 145 & 46 & 191 \\
Colecistectomia por minilaparotomia & 46 & 14 & 60 \\
\hline \multirow{2}{*}{ TOTAL } & $\mathbf{4 0 5}$ & $\mathbf{1 5 2}$ & $\mathbf{5 5 7}$ \\
& $\mathbf{( 7 2 , 7 \% )}$ & $\mathbf{( 2 7 , 3 \% )}$ & $\mathbf{( 1 0 0 \% )}$ \\
\hline
\end{tabular}

Todos os pacientes submetidos à coledocotomia tiveram sua via biliar drenada com Kher, sendo a drenagem da cavidade opcional. Quando a opção foi anastomose biliodigestiva, deu-se preferência à derivação coledocojejunal em Y-de-Roux, que foi feita em cinco pacientes. Em três realizou-se anastomose coledocoduodenal.

Colangiografia intra-operatória não foi realizada rotineiramente, mas nos pacientes que tiveram icterícia prévia, pancreatite, alterações das enzimas hepáticas, evidências de dilatação do ducto cístico ou colédoco, tanto no intra como no pré-operatório.

A Tabela 2 mostra o período de internação após as operações nas três modalidades. Observa-se que nos pacientes idosos houve sempre maior período de internamento, de modo especial no procedimento laparotômico, com média maior de internamento de 1,3 dias.

A Tabela 3 mostra o tempo operatório nas três modalidades de operação. Observa-se que não houve diferença entre os grupos.
TABELA 2 - Tempo de permanência hospitalar (em dias) após a operação

\begin{tabular}{lcc}
\hline Tipo de procedimento & Idade $<\mathbf{6 0}$ & Idade $\geq \mathbf{6 0}$ \\
\hline Colecistectomia por laparotomia & $\begin{array}{c}2-7 \\
(\mathrm{~m}=3,2)\end{array}$ & $\begin{array}{c}2-35 \\
(\mathrm{~m}=4,5)\end{array}$ \\
Colecistectomia por minilaparotomia & $1-2$ & $1-3$ \\
Colecistectomia videolaparoscópica & $\begin{array}{c}1-3 \\
(\mathrm{~m}=1,2)\end{array}$ & $\begin{array}{c}1-7 \\
(\mathrm{~m}=1,6)\end{array}$ \\
\hline
\end{tabular}

TABELA 3 - Tempo operatório (em minutos) nas três modalidades de colecistectomia

\begin{tabular}{lcc}
\hline Tipo de procedimento & Idade $<\mathbf{6 0}$ & Idade $\geq \mathbf{6 0}$ \\
\hline Colecistectomia laparotômica & $20-270$ & $20-240$ \\
Colecistectomia por minilaparotomia & $18-110$ & $18-120$ \\
Colecistectomia por laparoscopia & $16-150$ & $20-130$ \\
\hline
\end{tabular}

A Tabela 4 mostra as complicações, tanto per como pós-operatórias, relacionadas ao tipo de procedimento e a idade dos pacientes. Pode ser observado que o número de complicações e a gravidade foram maiores nas laparotomias e nos pacientes acima de 60 anos, assim como a mortalidade que só aconteceu nesta faixa etária.

TABELA 4 - Correlação das complicações quanto ao tipo de procedimento e a idade

\begin{tabular}{|c|c|c|}
\hline $\begin{array}{l}\text { Tipo de } \\
\text { procedimento }\end{array}$ & Idade $<60$ & Idade $\geq 60$ \\
\hline $\begin{array}{l}\text { Colecistectomia } \\
\text { laparotômica }\end{array}$ & $\begin{array}{l}\text {-lesão via biliar principal }=1 \\
\text { - fístula biliar }=2 \\
\text {-infecção urinária }=0 \\
\text { • infecção parede }=2 \\
\text { • corpo estranho na cavidade }=2\end{array}$ & $\begin{array}{l}\text {-lesão via biliar principal }=1 \\
\text {-fístula biliar }=3 \\
\text {-infecção urinária }=2 \\
\text { •infecção parede }=4 \\
\text { •óbito }=2\end{array}$ \\
\hline $\begin{array}{l}\text { Colecistectomia por } \\
\text { minilaparotomia }\end{array}$ & •lesão parcial do colédoco $=1$ & $\cdot$ pneumonia $=1$ \\
\hline $\begin{array}{l}\text { Colecistectomia por } \\
\text { laparoscopia }\end{array}$ & $\begin{array}{l}\text { - perfuração da vesícula biliar }=7 \\
\text { - sangramento do leito vesicular }=2 \\
\text { - fistula biliar }=1 \\
\text { - conversão }=6 \\
\text { - lesão parcial do hepático comum } \\
=1 \\
\text { - atelectasia pulmonar }=1\end{array}$ & $\begin{array}{l}\text {-perfuração da vesícula biliar }=5 \\
\cdot \text {-sangramento do leito vesicular }=2 \\
\cdot \text { fístula biliar }=1 \\
\cdot \text { conversão }=3 \\
\cdot \text {-hemoperitôneo }=1 \\
\cdot \text {-ICC }=1 \\
\cdot \text { óbito }=1\end{array}$ \\
\hline
\end{tabular}

O primeiro óbito ocorreu em uma paciente de 87 anos com colecistite aguda grave e coledocolitíase. Houve lesão inadvertida do colédoco, sendo necessária a realização de uma derivação bileodigestiva. Ela morreu de septicemia no $7^{\circ}$ dia do pós-operatório. O outro caso de óbito aconteceu também em uma paciente idosa (74 anos), com choque cardiogênico no $3^{\circ}$ dia do pós-operatório de colecistite aguda.

Nas intervenções realizadas por minilaparotomia, as duas lesões mais graves ocorreram em pacientes jovens e tiveram boa evolução. O único caso de infecção respiratória em paciente idoso também evoluiu bem.

Quanto às colecistectomias videolaparoscópicas, observaram-se complicações, de um modo geral, semelhantes nos dois grupos de pacientes. Todavia, a mais séria foi em paciente de 76 anos, cuja operação transcorreu sem intercorrências. Após 24 horas do procedimento a paciente apresentou dor abdominal contínua, difusa, sendo 
reoperada após 36 horas. O achado foi coleperitônio por ducto aberrante, que foi suturado e a cavidade abdominal limpa com soro fisiológico e drenada. No pós-operatório evoluiu com importante desequilíbrio hidroeletrolítico, insuficiência renal aguda e óbito.

\section{DISCUSSÃO}

Descrita inicialmente em 1987, a colecistectomia laparoscópica é considerada hoje o tratamento de escolha para a colecistolitíase $e^{4,8}$, pois apresenta índice de mortalidade e morbidade semelhantes à colecistectomia laparotômica, com a vantagem de permitir retorno precoce às atividades sociais e ao trabalho, menor tempo de permanência hospitalar e menor agressão cirúrgica ${ }^{1,9}$.

Embora a via laparoscópica possa ser usada em qualquer faixa etária, diferentemente de quando se iniciou o seu aprendizado - época que não era indicada em pacientes acima de 65 anos -, seu índice de complicações e mortalidade não difere do procedimento laparotômico.

Nesta casuística pôde-se observar complicações nas várias modalidades, sendo as mais graves os três óbitos $(0,53 \%)$. A mortalidade relacionada à colecistectomia ocorre com freqüência aproximada de $0,2 \%$ e é aumentada em aproximadamente cinco vezes quando se explora a via biliar principal $(0,2$ a $1,0 \%)$. Quando se realiza procedimento de drenagem biliar, a mortalidade pode atingir $2 \%$ a $5 \%{ }^{10,11,12}$. A mortalidade depende também da idade, sendo quase nula nos indivíduos abaixo de $60 \operatorname{anos}^{11,12,13}$.

Os três óbitos ocorridos neste estudo foram em pacientes idosos - 74, 76 e 87 anos -, todos do sexo feminino, dado que é discrepante da literatura, pois a tendência é de a mortalidade e outras complicações apresentarem freqüências maiores nos homens, apesar do predomínio da doença e do tratamento cirúrgico no grupo feminino ${ }^{14}$.

As duas pacientes que foram a óbito após colecistectomia por via laparotômica, apresentavam-se com doença complicada (colecistite aguda), sendo que a de menor idade (74 anos) tinha como co-morbidade apenas hipertensão arterial e desenvolveu choque cardiogênico no $3^{\circ}$ dia de pós-operatório, cuja causa exata não pôde ser determinada. A paciente de 87 anos além de colecistite aguda, apresentava icterícia obstrutiva por coledocolitíase e durante o intra-operatório houve lesão inadvertida do colédoco, sendo necessário a ampliação do ato cirúrgico, através de derivação biliodigestiva. O óbito ocorreu no $7^{\circ}$ dia de pósoperatório decorrente de septicemia.

A paciente de 76 anos submetida à colecistectomia videolaparoscópica não apresentava complicação prévia de sua doença e tinha como co-morbidade apenas hipertensão arterial. O óbito aconteceu por desequilíbrio hidroeletrolítico e insuficiência renal aguda, decorrente de coleperitônio por ducto aberrante, sendo reoperada após 36 horas. Chamou a atenção neste caso a dor abdominal que persistia após 12 horas da operação, fato incomum nas colecistectomias videolaparoscópicas. A indicação para nova operação aconteceu quando ocorreram sinais importantes de irritação peritonial e em momento onde a paciente já apresentava alterações eletrolíticas e insuficiência pré-renal. É possível que uma intervenção mais precoce trouxesse benefícios.

A mortalidade da colecistectomia laparoscópica é inferior a $1 \%{ }^{16,17}$. Letalidade mais elevada é descrita na literatura em relação à população acima de 50 anos. O percentual de óbito descrito no estudo de Golden et al. ${ }^{18}$ que incluiu apenas pacientes com idade superior a 65 anos foi de 1,3\%, mas outras constatações chegam a índices de $10 \%{ }^{14}$.

Os casos de infecção da ferida cirúrgica, urinária e respiratória, têm ocorrido com freqüência muito baixa no serviço dos autores ${ }^{19,20,21}$, decorrente provavelmente de grande preocupação que se tem tido com este assunto, fazendo com que o uso de antimicrobianos seja criterioso, além da existência de comissão de controle de infecção hospitalar atuante e realiza busca ativa constante. Todavia pode-se observar que tanto a infecção urinária como a do sitio cirúrgico foram mais freqüentes nos idosos e só ocorreram nas operações laparotômicas. Se for considerada somente esta faixa etária, observou-se que o índice de infecção urinária foi de 2,1\% (dois casos em 92 intervenções) e do sitio cirúrgico de 4,3\% (quatro casos em 92), valores superiores aos apresentados nas faixas etárias mais baixas.

Nesta casuística observaram-se quatro casos de lesão de via biliar principal, sendo três em doentes abaixo de 60 anos e um acima desta faixa etária. Este último teve má evolução, o que não ocorreu com os demais. Paciente idoso com quadro de colecistite aguda é situação que reconhecidamente aumenta o risco de lesão iatrogênica da via biliar ${ }^{22}$.

A incidência de lesão dessa via neste estudo foi de $0,7 \%$, que está de acordo com estudo nacional que observou esta complicação em $0,9 \%$ e é acima dos relatados em outros países, mas talvez não se possa comparar casuísticas em condições sociais tão diversas ${ }^{23,24}$.

O tempo cirúrgico médio para as colecistectomias laparotômicas e laparoscópicas foram, de um modo geral semelhantes entre idosos e mais jovens, e menores ou iguais a outros relatos da literatura ${ }^{4,14}$. Já o período de internação foi maior nos pacientes idosos submetidos à laparotomia. Certamente a permanência hospitalar mais prolongada nos pacientes idosos, está relacionada ao número maior de complicações nesta faixa etária.

Ficou evidente que este grupo de pacientes requer cuidados especiais no seu preparo e nos cuidados pósoperatórios, e certamente se beneficiam com cuidados mutiprofissionais

\section{CONCLUSÃO}

A idade é importante fator preditivo de complicações após a colecistectomia tanto laparotômica como laparoscópica, devido à incidência progressivamente aumentada da doença da via biliar associada com a idade e co-morbidades normalmente existentes nos idosos. 
Minossi JG, Picanço HC, Carvalho MA, Paulucci PRV, Vendites S. Morbity and mortality of elderly patients submitted to cholecystectomy by laparotomy, minilaparotomy or videolaparoscopy. ABCD Arq Bras Cir Dig 2007; 20(2):93-6.

ABSTRACT - Background - Cholelithiasis is one of the most frequent diseases regarding the digestive system. It is present in about $20 \%$ of the adult population, being age an important predictive factor for complications after cholecystectomy. Aim - To evaluate the morbimortality rates concerning elderly patients who underwent cholecystectomy and who are submitted to conventional practices, minilaparotomy and laparoscopy. Methods - Five hundred and fifty seven patients submitted to cholecystectomy, associated or not to other procedures on the biliary tract, were observed during the period of July 1985 and December 2003. One hundred and fifty two $(27,3 \%)$ were 60 years or over and $120(79 \%)$ were female. Ninety two were submitted to conventional practices, 46 to laparoscopy and 14 to minilaparotomy. Results - Complications were frequent and serious in elder patients and the ones submitted to conventional practices presented greater incidence of urinary infection, surgical wound infection and a longer time of hospitalization. There were three obits, two of them after conventional surgery and the other after videolaparoscopic surgery. All of the patients were over 70 years old. Conclusion - It was possible to conclude that age is an important predictive factor of complications after cholecystectomy as a result of the increase in the incidence of biliary tract disease as well as because of a greater morbidity and mortality in elderly patients resulting from associated diseases.

HEADINGS - Cholecystectomy. Laparoscopy. Cholelithiasis. Mortality.

\section{REFERÊNCIAS}

1. Bergman JJ, Van Den Brink GR, Raws EA. Treatment of bile duct lesion after laparoscopic cholecystectomy. Gut. 1996;38:141-7.

2. Csendes A, Burdiles P, Diaz JC. Present role of classic open choledochostomy in the surgical treatment of patients with common bile duct stones. World J Surg. 1998;22:167-70.

3. De Wit LT, Raws AJ, Gouma DJ. Surgical management of bile duct injury. Scand J Gastroenterol. 1999;34 (Suppl 230):89-94.

4. Fiorillo MA, Davidson PG, Fiorillo M, et al. 149 ambulatory laparoscopic cholecystectomies. Surg Endosc. 1998;10:52-6.

5. Goco IR, Chambers LG. Dollar and cents: mini-cholecystectomy and early discharge. South Med J. 1998;81:162-3.

6. Golden WE, Cleves MA, Johnston JC. Laparoscopic cholecystectomy in the geriatric population. J Am Geriatr Soc. 1996;44:1380-3.

7. Haicken BN. Laser laparoscopic cholecistectomy in the ambulatory setting. J Post Anesth Nurs. 1991;6:33-9.

8. Hangui RM, Rêgo RE, Demarchi VC, Tomasich FD, Pacheco Jr AM. Complicações pós-operatórias de colecistectomias - análise comparativa em relação ao sexo. Rev Col Bras Cir. 2004;31:57-63.

9. Henriques AC, Pezzolo S, Gomes M, Godinho CA, Bagarolho CA. Colecistectomia videolaparoscópica ambulatorial. Rev Col Bras Cir. 2000;28:27-9.

10. Kumar A, Saxena R, Kapoor VK et al. Cholecystectomy in high risk patients. Trop Gastroenterol. 1996;17:53-6.

11. Langenbuch C. Ein fall extirpation der gallenblase wegen chronischer cholelithiasis. Heinbung Klein Wschr. 1882;48:725-7 .

12. McSherry CK. Advantages of elective surgical treatment of gallstones. Hepatogastroenterology. 1989;36:330-2.

13. Minossi JG, Pedro FAJ, Vendites S, Santos CF. Experiência de um serviço de cirurgia em um pequeno hospital o interior de São Paulo. J Bras Med. 1990;59:76-88.
14. Minossi JG, Pedro FA, Santos CF, Vendites S. Influência do antibiótico profilático na morbidade febril puerperal pós-cesariana. J Bras Gastroenterol 1993;103:159-62.

15. Minossi JG, Spadella CT, Picanço HC, Paulucci PR, Vendites S. Experiência de um serviço de cirurgia geral em um hospital do interior do estado de São Paulo. Estudo de 4022 casos. Anais do XXIX Congresso Brasileiro de Cirurgia. São Paulo; 2001.

16. Moss C. Discharge within 24 hours of elective cholecystectomy. Arch Surg. 1996;121:155-61.

17. Neves CC, Penteado S, Zilberstein B, Neves G, Gabriel Neto S, Oliveira EC Incidência e resultado do tratamento das lesões da via biliar em colecistectomia por laparotomia. ABCD Arq Bras Cir Dig. 2004;17:38-41.

18. Orlando R, Russell JC, Lynch J, et al. Laparoscopic cholecystectomy. A statewide experience. The Connecticul laparoscopic cholecystectomy registry. Arch Surg. 1993;128:494-8.

19. Ramos AC, Sallet JA, Lombardi MM. Colecistectomia laparoscópica em regime ambulatorial. Rev Col Bras Cir. 1999;25:5-8.

20. Rosenthal RJ, Rossi RF, Martin RF. Options and strategies for the management of choledocholithiasis. World J Surg. 1998;22:125-32.

21. Russel JC, Walsh SJ, Reed-Fourguet, et al. Syntomatic cholelithiasis: a different disease in men? Connecticut laparoscopic cholecystectomy registry. Ann Surg. 1998;227:195-200.

22. Silva RA, Rasslan S. Análise das indicações e resultados de coledocotomia, papiloesfincterostomia e derivações bíleo-digestivas no tratamento convencional da coledocolitíase. In: De Paula A, Hashiba K, Bafutto Meds, editores. Cirurgia videolaparoscópica. Goiânia: Edição particular; 1994. p.213.

23. Soper NJ, Stockmann PT, Dunnegan DL, et al. Laparoscopic cholecystectomy. The new "gold standard"? Arch Surg. 1992;127:917-21.

24. Strom PR, Stone HH. A technique for transduodenal sphincteroplasty. Surgery. 1982;92:546-9.

Conflito de interesse: não há

Fonte financiadora: não há

Recebido para publicação em: 03/01/2007 Aceito para publicação em: 13/04/2007 